\title{
Measurement Reliability for Evaluating Lumbopelvic Curvature during Active Knee Extension in Sitting Using a Flexible Ruler in Individuals with Clinically Tight Hamstring Muscles: A Secondary Analysis
}

\author{
Hiroshi Takasaki1 ${ }^{*}$, Kazuki Kikkawa1, Toby Hall² \\ ${ }^{1}$ Department of Physical Therapy, Saitama Prefectural University, Koshigaya, Japan \\ ${ }^{2}$ School of Physiotherapy and Exercise Science, Curtin University, Perth, Australia \\ Email: ^takasaki-hiroshi@spu.ac.jp
}

How to cite this paper: Takasaki, H., Kikkawa, K. and Hall, T. (2018) Measurement Reliability for Evaluating Lumbopelvic Curvature during Active Knee Extension in Sitting Using a Flexible Ruler in Individuals with Clinically Tight Hamstring Muscles: A Secondary Analysis. Open Journal of Therapy and Rehabilitation, 6, 1-7. https://doi.org/10.4236/ojtr.2018.61001

Received: December 17, 2017 Accepted: February 6, 2018

Published: February 9, 2018

Copyright $\odot 2018$ by authors and Scientific Research Publishing Inc. This work is licensed under the Creative Commons Attribution International License (CC BY 4.0).

http://creativecommons.org/licenses/by/4.0/

\begin{abstract}
In a previous study, we established reliability of a method for determining the angle of lumbopelvic sagittal alignment during active knee extension in sitting (AKEiS) using a flexible ruler and image analysis software (2-point-Method). In addition to this method, a flexible ruler can also be used to measure lumbopelvic sagittal alignment without image analysis software. This study primarily aimed to investigate the minimum number of repetitions, inter-session reliability and inter-examiner reliability of two alternative methods of measurement in a secondary analysis of our previous study. A flexible ruler was used to measure lumbopelvic curvature during AKEiS when the knee reached $10^{\circ}$ flexion from 27 individuals with clinically tight hamstring muscles and subsequently analyzed. Lumbopelvic sagittal alignment was evaluated for the region between T12 and S2 using the maximum depth to the curvature (Max-Method) or depth to the curvature at the middle point between T12 and S2 vertebral levels (Mid-Method). It was determined that four repetitions for the Max-Method and 11 repetitions for the Mid-Method were required for the minimum number of repetitions, respectively. Inter-session reliability and inter-examiner reliability were assessed using Intraclass Correlation Coefficients and were 0.91 and 0.91 for the Max-Method and 0.90 and 0.91 for the Mid-Method, respectively. The current study suggests that the Mid-Method would not be recommended for use in the clinical setting as 11 repetitions of data sampling is required. The 2-point-Method or Max-Method may be promising but the ideal measurement method will be identified when the va-
\end{abstract}


lidity of these methods has been established.

\section{Keywords}

lumbosacral Region, Muscle Tightness, Hamstring, Physical Examination, Relative Flexibility, Test-Retest Reliability

\section{Introduction}

Relative flexibility between the lumbar spine and the hamstring muscles has been considered an important factor in the physical evaluation of people with low back pain (LBP) [1] [2] [3]. Evaluating the effect of hamstring muscles tightness on lumbopelvic curvature can be determined by the active knee extension test in sitting (AKEiS), and is recommended to be used in the assessment of LBP [4].

We recently reported a reliable and quantitative method of measuring lumbopelvic curvature during AKEiS using a flexible ruler and Image J software by considering the minimum number of repetitions, as well as inter-session reliability and inter-examiner reliability [5]. In this method (2-point-Method), lumbopelvic curvature was traced from T12 to S2 spinous process using a flexible ruler, where a tangential line on the traced line of the lumbopelvic curvature at T12 and S2 vertebral levels were drawn and the angle between the two tangential lines calculated. A previous study [6] used Auto CAD software to draw a vertical line on the traced line of the lumbar curvature at T12 and S2 vertebral level and demonstrated high correlation between the angle calculated and the cobb angle on the $\mathrm{x}$-ray (Intraclass Correlation Coefficient $[$ ICC $]=0.94-0.96$ ). Thus, criterion-related validity of the 2 -point-Method is assumed.

In addition to the 2-point-Method, there are two possible measurement methods for the assessment of lumbopelvic sagittal alignment using the flexible ruler. A feature of both these methods (in contrast to the 2-point-Method) is convenience due to the lack of the need for Image J software. Both methods consider the length between the T12 and S2 vertebral levels $(L)$ and depth to the curvature $(H)$ and calculate the angle of lumbopelvic sagittal alignment $(\theta)$ using the following formula:

$$
\theta=4 \operatorname{Arctan} \frac{2 H}{L}
$$

A difference between the two methods is the definition of the depth to the curvature. In one method, the maximum depth to the curvature is used (Max-Method). In the other method, the depth to the curvature at the middle point between the T12 and S2 vertebral level is used (Mid-Method).

A previous study [6] demonstrated that the ICC for the angle calculated with the Mid-Method when compared to the $\mathrm{x}$-ray determined cobb angle was 0.51 0.52. Inter-session reliability and inter-examiner reliability of the Mid-Method were $0.62-0.69$ and 0.55 , respectively. However, the number of repetitions of 
the measure was not reported in that study [6]. Another study [7] reported that the angle calculated with the Mid-Method was more consistent with the $\mathrm{x}$-ray determined cobb angle than the angle calculated using the Max-Method. However, reliability of the Max-Method has not been comprehensively evaluated. Further, it is unknown if the angles calculated with the 2-point-Method, the Max-Method and the Mid-Method correlate with each other.

The current study was a secondary analysis of data from our previous study. The primary aim was to investigate the minimum number of repetitions, and inter-session and inter-examiner reliability of the Max-Method and Mid-Method for measuring lumbopelvic sagittal alignment. The secondary aim was to investigate the correlation between the three measurement methods, the method used in the previous study and two new methods using a flexible ruler.

\section{Methods}

\subsection{Design}

This is a secondary analysis of our previous study [5]. Ethical approval was obtained from the Research Ethics Committee of the Saitama Prefectural University. Written consent was obtained from each participant prior to data collection.

\subsection{Data Source}

The current study analyzed data from 27 participants ( 14 men and 13 women, mean \pm SD years of age $=21.1 \pm 2.8$ years) with clinically tight hamstring muscles [5], who developed kyphosis of the lower back during the right AKEiS [4] [8]. Specific inclusion and exclusion criteria have been previously described [5]. Briefly, the participants neither had lower limb symptoms or limitation of hip, knee or ankle range of motion, nor scoliosis.

Detailed procedures of data acquisition were described in the previous study [5]. Briefly, during the AKEiS when the right knee achieved $10^{\circ}$ flexion with the foot/ankle held in full plantar flexion, lumbopelvic curvature was traced from T12 to S2 spinous process using a flexible ruler (Shinwa Rules Co., Ltd., Tsubame, Niigata, Japan). The end-point of $10^{\circ}$ knee flexion was standardized using a metal orthosis. Data were collected on 15 repeated trials by two examiners. For each participant, the angle of lumbopelvic sagittal alignment was calculated by two examiners, whose results were blinded to each other throughout data analysis. To investigate inter-session reliability, data were again collected on 15 repeated trials on consecutive days at a similar time of a day ( \pm three hours) by one examiner.

\subsection{Measurements}

Detailed information for the 2-point-Method was described in the previous study [5]. Briefly, tangential lines on the traced line of the lumbopelvic curvature at T12 and S2 vertebral levels were drawn with Image J1.6 software (National Institute of Mental Health, Bethesda, MD, USA). The angle between the two tan- 
gential lines was calculated, where a positive value indicated lumbopelvic kyphosis and a negative value indicated lumbopelvic lordosis.

Regarding the Max-Method and the Mid-Method, the distance between T12 and S2 vertebral levels $(L)$ and depth to the curvature $(H)$ were measured on the traced line of the lumbopelvic curvature. Then, the angle of lumbopelvic sagittal alignment $(\theta)$ was calculated using the formula described above. Again, a positive value indicated lumbopelvic kyphosis and a negative value indicated lumbopelvic lordosis.

\subsection{Statistics}

The minimum number of repetitions was investigated with data collected by one examiner at the first session using the Max-Method and the Mid-Method. Detailed procedures of analysis were described previously [5]. Briefly, means for the angle were calculated using an increasing number of successive repetitions from 2 to 15 , and then the absolute difference in mean value with successive inclusion of additional repetitions were calculated [9] [10]. The minimum number of repetitions was defined as the largest number of repetitions, where the $P$-value of the paired sample t-test between $\mathrm{N}-1$ and $\mathrm{N}$ repetitions $(\mathrm{N}=2$ to 15$)$ was $<0.05$. For subsequent analyses, a representative value of the angle for each method was calculated with the identified minimum number of repetitions.

Subsequently, inter-session and inter-examiner reliability was assessed with ICC for the Max-Method and Mid-Method. Further, the minimum detectable change (MDC) was calculated with inter-session data using the following standard formula;

$$
\begin{gathered}
\mathrm{MDC}=\mathrm{SEM} \times 1.96 \times \sqrt{2} \\
\mathrm{SEM}=\mathrm{SD} \sqrt{1-(\text { ICC } \text { of Inter-session Reliability })}
\end{gathered}
$$

Correlations of the calculated angle between the three methods were evaluated with ICC. Interpretations of the ICC values were as follows: poor reliability, ICC $<0.4$; fair-good reliability, ICC $=0.4$ - 0.75; excellent reliability, ICC > 0.75 [11].

SPSS (IBM Corporation, Armonk, NY, USA) was used for statistical analyses. The level of statistical significance was set at $5 \%$.

\section{Results}

In relation to the Max-Method, Figure 1 demonstrates the individual and group mean absolute differences between the mean value of $\mathrm{N}-1$ and $\mathrm{N}$ repetitions ( $\mathrm{N}$ $=2$ to 15) for the angle of lumbopelvic sagittal alignment. There was no statistical difference $(P>0.05)$ between the mean value of $\mathrm{N}-1$ and $\mathrm{N}$ repetitions $(\mathrm{N}=2$ to 3 , and 5 to 15 ) and therefore four was considered the minimum number of repetitions. The $\operatorname{ICC}_{(1,4)}(95 \%$ confidence intervals) for inter-session reliability and $\operatorname{ICC}_{(2,4)}$ for inter-examiner reliability was $0.91(0.81-0.96)$ and $0.91(0.79-0.96)$, respectively. These ICC values indicate excellent inter-session reliability and inter-examiner reliability for these measurement methods. The MDC was $11.28^{\circ}$. 


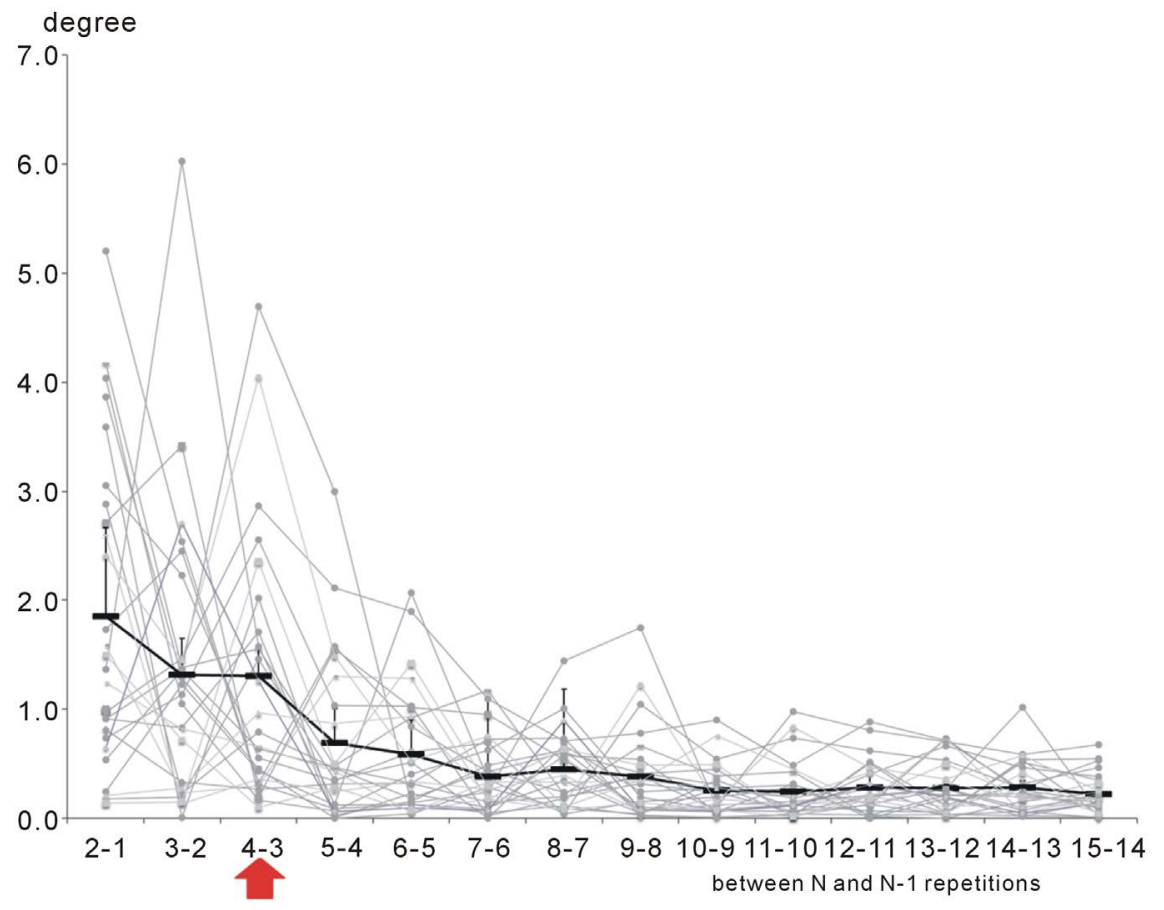

Figure 1. Difference in the absolute difference between the mean value of $\mathrm{N}$ repetitions and $\mathrm{N}-1$ repetitions $(\mathrm{N}=2$ to 15$)$ for the angle of lumbopelvic sagittal alignment with the Max-Method. A red arrow indicates that there is a statistically significant difference $(\mathrm{P}<0.05)$ between the mean value of three and four repetitions.

In relation to the Mid-Method, Figure 2 demonstrates the individual and group mean absolute differences between the mean value of $\mathrm{N}-1$ and $\mathrm{N}$ repetitions ( $\mathrm{N}=2$ to 15$)$ for the angle of lumbopelvic sagittal alignment. There was no statistical difference $(P>0.05)$ between the mean value of $\mathrm{N}-1$ and $\mathrm{N}$ repetitions $(\mathrm{N}=2$ to 9 , and 12 to 15$)$ and therefore 11 was considered the minimum number of repetitions. The $\mathrm{ICC}_{(1,11)}$ for inter-session reliability and $\mathrm{ICC}_{(2,11)}$ for inter-examiner reliability was $0.90(0.79-0.96)$ and $0.91(0.79-0.96)$, respectively. These ICC values indicate excellent inter-session reliability and inter-examiner reliability. The MDC was $12.17^{\circ}$.

Table 1 presents correlations between the three methods. Fair-good reliability was seen between the 2-point-Method and the Max-Method or the Mid-Method and excellent reliability was seen between the Max-Method and the Mid-Method.

\section{Discussion}

The current secondary analysis of our previous study [5] demonstrated acceptable inter-session reliability and inter-examiner reliability for either Max-Method or Mid-Method for the angle of lumbopelvic sagittal alignment. However, the Mid-Method required 11 repetitions of data sampling whereas the Max-Method and the 2-point-Method required four and five repetitions of data sampling, respectively. Therefore, the Mid-Method would not be recommended to be used in the clinical setting unless criterion-related validity to the $\mathrm{x}$-ray results is found only in the Mid-Method. 


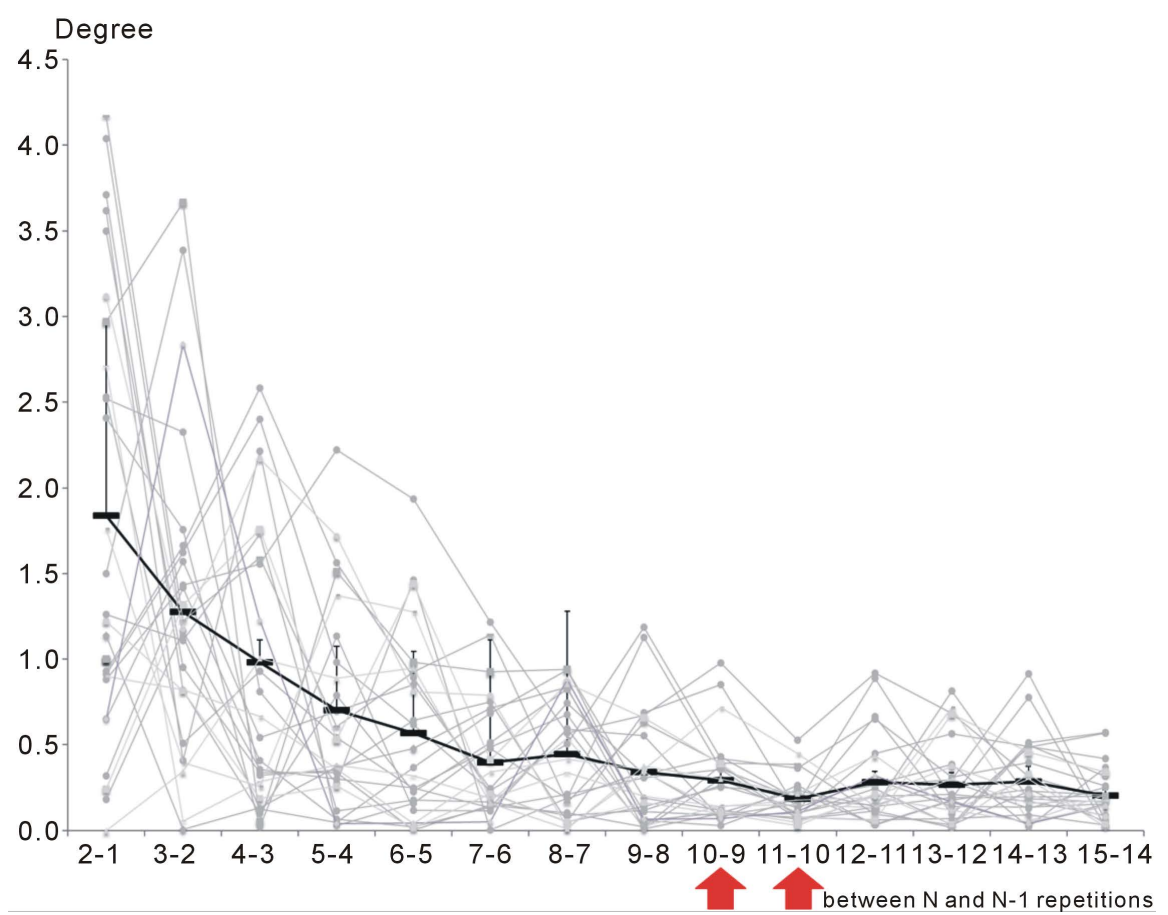

Figure 2. Difference in the absolute difference between the mean value of $\mathrm{N}$ repetitions and $\mathrm{N}-1$ repetitions ( $\mathrm{N}=2$ to 15$)$ for the angle of lumbopelvic sagittal alignment with the Mid-Method. Red arrows indicate that there are statistically significant difference $(\mathrm{P}<$ $0.05)$ between the mean value of $\mathrm{N}-1$ and $\mathrm{N}$ repetitions $(\mathrm{N}=9$ and 10).

Table 1. Intraclass correlation coefficients (95\% confidence intervals) [p-value] between three methods of measurement for the angle of lumbopelvic sagittal alignment.

\begin{tabular}{ccc}
\hline & Max-Method & Mid-Method \\
\hline 2-point-Method & $0.58(0.09-0.81)$ & $0.66(0.27-0.85)$ \\
& {$[0.014]$} & {$[0.003]$} \\
Max-Method & $0.91(0.81-0.96)$ \\
& & {$[<0.001]$} \\
\hline
\end{tabular}

Fair-good reliability was seen between the 2-point-Method and the Max-Method. This finding indicates the Max-Method may be used as an alternative to the 2-point-Method. The Max-Method is more user friendly than the 2-point-Method due to the lack of analysis with image software. Investigation of validity will be required to identify the best method for measurement of the angle of lumbopelvic sagittal alignment using a flexible ruler.

\section{Conclusion}

The current secondary analysis of our previous study [5] found acceptable inter-session reliability and inter-examiner reliability for either Max-Method or Mid-Method for the angle of lumbopelvic sagittal alignment. However, the Mid-Method would not be recommended for use in clinical settings as 11 repetitions of data sampling is required. 


\section{Acknowledgements}

The authors acknowledge Mr. Musashi Yasuda and Ms. Kana Nishimoto for assistance of data analysis.

\section{References}

[1] Gombatto, S.P., Collins, D.R., Sahrmann, S.A., Engsberg, J.R. and Van Dillen, L.R. (2007) Patterns of Lumbar Region Movement during Trunk Lateral Bending in 2 Subgroups of People with Low Back Pain. Physical Therapy, 87, 441-454. https://doi.org/10.2522/ptj.20050370

[2] Kim, M., Yoo, W. and Choi, B. (2013) Differences between Two Subgroups of Low Back Pain Patients in Lumbopelvic Rotation and Symmetry in the Erector Spinae and Hamstring Muscles during Trunk Flexion when Standing. Journal of Electromyography \& Kinesiology, 23, 387-393.

https://doi.org/10.1016/j.jelekin.2012.11.010

[3] Harris-Hayes, M. and Van Dillen, L.R. (2009) The Inter-Tester Reliability of Physical Therapists Classifying Low Back Pain Problems Based on the Movement System Impairment Classification System. Journal of the American Academy of Physical Medicine and Rehabilitation, 1, 117-126.

[4] Sahrmann, S.A. (2001) Diagnosis and Treatment of Movement Impairment Syndromes. Mosby, St Louis.

[5] Yasuda, M., Nishimoto, K., Hori, M., Noguchi, T. and Takasaki, H. (2017) The Effect of Active Knee Extension in Sitting on Lumbopelvic Curvature in Individuals with Clinically Tight Hamstring Muscles: A Cross-Sectional Reliability Study. Open Journal of Therapy and Rehabilitation, 5, 139-147. https://doi.org/10.4236/ojtr.2017.54012

[6] Eslam, B., Aslan, K., Zahra, M. and Saleh, B. (2012) An Innovative Software Method for Measuring Lumbar Lordosis. Annals of Biological Research, 3, 204-213.

[7] Rajabi, R., Seidi, F. and Mohamadi, F. (2008) Which Method Is Accurate When Using the Flexible Ruler to Measure the Lumbar Curvature Angle? Deep Pint or Mid Point of Arch? World Applied Sciences Journal, 4, 849-852.

[8] Kendall, F.P., McCreary, E.K., Provance, P.G., Rodgers, M.M. and Romani, W.A. (2005) Muscles, Testing and Function with Posture and Pain. Lippincott Williams \& Wilkins, Baltimore.

[9] Swait, G., Rushton, A.B., Miall, R.C. and Newell, D. (2007) Evaluation of Cervical Proprioceptive Function: Optimizing Protocols and Comparison between Tests in Normal Subjects. Spine, 32, E692-E701. https://doi.org/10.1097/BRS.0b013e31815a5a1b

[10] Takasaki, H., Treleaven, J., Johnston, V. and Jull, G. (2012) Minimum Repetitions for Stable Measures of Visual Dependency Using the Dot Version of the Computer-Based Rod-Frame Test. Manual Therapy, 17, 466-469. https://doi.org/10.1016/j.math.2012.02.013

[11] Fleiss, J.L. (1986) The Design and Analysis of Clinical Experiments. John Wiley and Sons, New York. 